\title{
Variación en la abundancia de Artropoda en un transecto latitudinal del desierto costero transicional de Chile, con énfasis en los tenebriónidos epígeos
}

\author{
Variation in the abundance of Arthropoda from a latitudinal transect in the transitional \\ coastal desert of Chile, with emphasis on the epigean tenebrionids
}

JORGE CEPEDA-PIZARRO*, JAIME PIZARRO-ARAYA \& HERNÁN VÁSQUEZ

Laboratorio de Entomología Ecológica, Departamento de Biología, Universidad de La Serena, La Serena, Chile; *e-mail para correspondencia: jcepeda@userena.cl

\begin{abstract}
RESUMEN
Mediante el empleo de trampas de intercepción se examinó la composición taxonómica y los patrones de abundancia (denso-actividad) de la fauna de Arthropoda del transecto $27-30^{\circ} \mathrm{S}$ del desierto costero transicional de Chile, particularmente la distribución geográfica y las relaciones de abundancia de los componentes del ensamble de Tenebrionidae. Durante el período estudiado, cinco órdenes dominaron numéricamente el ensamble de Arthropoda. Estos fueron Coleoptera, Diptera, Psocoptera, Collembola e Hymenoptera. Representada por 14 géneros, Tenebrionidae fue la familia más diversa y abundante de la fauna de artrópodos epígeos. Gyriosomus Guérin-Méneville, 1834 fue el género más diverso de la familia. Algunas de sus especies mostraron una distribución restringida en el área estudiada y podrían ser indicadoras de endemismo. La riqueza de especies de Tenebrionidae no siguió el patrón pluviométrico latitudinal del transecto. Carrizal Bajo, un sitio con características xéricas, aportó un número mayor que el esperado, en concordancia con lo mostrado por la flora, particularmente geófitas y hemicriptófitas.
\end{abstract}

Palabras clave: zonas áridas, desierto costero, diversidad biológica, artrópodos epígeos, Tenebrionidae, trampas de intercepción.

\begin{abstract}
By using pitfall traps and with special emphasis on the geographical distribution and the relationships of abundance of the Tenebrionidae assemblage, the taxonomic composition and the patterns of abundance (denso-activity) of the Arthropoda inhabiting the transect $27-30^{\circ} \mathrm{S}$ of the chilean transitional coastal-desert were examined. Five orders numerically dominated the Arthropoda assemblage. These were Coleoptera, Diptera, Psocoptera, Collembola and Hymenoptera. Tenebrionidae, represented by 14 genera, was the most diverse and abundant family of the epigean arthropod-fauna. Gyriosomus Guérin-Méneville, 1834 was the most specious genus in that family. Some of its species showed a narrow distribution in the study area, and may be indicators of endemisms. The species richness of Tenebrionidae did not follow the latitudinal pluviometric pattern of the transect. Carrizal Bajo, a site with xeric conditions, provided a higher number than expected, in accordance with the pattern shown by the flora, mainly geophytes and hemicryptophytes.
\end{abstract}

Key words: arid zones, coastal desert, biological diversity, epigean arthropods, Tenebrionidae, pitfall traps.

\section{INTRODUCCIÓN}

El papel que juegan los artrópodos en la estructura y función de los ecosistemas áridos de Chile, así como sus patrones de diversidad y abundancia, solo recientemente han comenzado a generar preguntas en los investigadores chilenos (Cepeda-Pizarro 1989, Marquet et al.
1998, Rau et al. 1998, Spotorno et al. 1998, Veloso \& Núñez 1998, Jerez 2000, CepedaPizarro et al. 2003, Carmona et al. 2003 ${ }^{1}$ ). De

${ }^{1}$ CARMONA C, A JORQUERA, J CEPEDA-PIZARRO \& H VÁSQUEZ (2003) Artropodofauna de un ecotopo dunario costero del Parque Nacional Llanos de Challe (III Región de Atacama). XXV Congreso Nacional de Entomología, Talca, Chile, Libro de Resúmenes: 34. 
estos trabajos surgen varias hipótesis, algunas de las cuales se enumeran a continuación: (1) la abundancia de los artrópodos de los ambientes desérticos del norte chileno disminuiría con la altitud; (2) la curva de distribución de la riqueza de sus especies sería unimodal, con valores máximos a altitudes intermedias; (3) existirían áreas de endemismo y diversidad específica; (4) la abundancia y diversidad estarían positivamente relacionadas con la diversidad de la vegetación; (5) la actividad fenológica sería marcadamente estacional, ligada a la disponibilidad y calidad del alimento, y (6) Tenebrionidae sería una de las familias más abundante y diversa de Arthropoda.

Tenebrionidae es una reconocida familia de la entomofauna de ecosistemas de desiertos (Peña 1959, Ahearn 1971, Crawford 1979, 1981, 1988a, 1988b, Wallwork 1982, Parmenter \& MacMahon 1984, 1988, de los Santos et al. 1988, 2000, 2002b, Parmenter et al. 1989a, 1989b, Crawford et al. 1993, Ayal \& Merkl 1994, Krasnov et al. 1996, Carpaneto \& Fattorini 2001, 2003). Se considera que estos insectos desempeñan un papel clave en los procesos de fragmentación biológica del recurso vegetal, en los ciclos de los nutrientes y en la dieta de otros organismos consumidores, particularmente vertebrados (Fortuna 1938, Peña 1959, Crawford 1981, 1988a, Wallwork 1982, Dajoz 1984, Sheldon \& Rogers 1984, Wise 1985, Parmenter \& MacMahon 1988, Díaz \& Díaz 1990, Flores 1998, Fattorini 2001, Semida et al. 2001, Adamopoulou \& Legakis 2002, Orgeas et al. 2003, Chen et al. 2004). Junto a lo anterior, algunas especies de Tenebrionidae se consideran posibles indicadores de condiciones climáticas (Marcuzzi 1951). Para el sector meridional de la franja $21-26^{\circ} \mathrm{S}$ del desierto costero chileno, Jerez (2000) ha reportado la presencia de siete géneros y 18 especies de Tenebrionidae, la mayoría de ellas endémicas al sector.

Además de constituir la región del desierto transicional (Rundel \& Mahu 1976, Rundel et al. 1991, Cepeda-Pizarro 1995), la franja 27$30^{\circ} \mathrm{S}$ del territorio chileno es el límite septentrional del foco de diversidad vegetal ("hotspots") reconocido para Chile Central (Cowling et al. 1996, Gaston 2000), y un área poco conocida en términos de su fauna de artrópodos (Cepeda-Pizarro 1987, 1989,
Cepeda-Pizarro et al. 2003, Carmona et al. $2003^{1}$, Pizarro-Araya \& Jerez 2004, PizarroAraya \& Flores 2004, Pizarro-Araya et al. $2004^{2}$ ). Antecedentes reportados en la literatura (Armesto \& Vidiella 1993, Armesto et al. 1993, Vidiella et al. 1999, Gutiérrez et al. 2000a) indican que la dinámica de la vegetación del área de estudio es simultáneamente agua y sitio-dependiente. En este trabajo postulamos que la dinámica del ensamble de artrópodos epígeos sigue el mismo patrón. Los objetivos del trabajo fueron: (1) documentar, a nivel ordinal, la composición taxonómica de Arthropoda y las variaciones espaciotemporales de su abundancia relativa; (2) describir la composición taxonómica de los tenebriónidos epígeos y las variaciones espacio-temporales de su abundancia relativa.

\section{MATERIALES Y MÉTODOS}

\section{Composición taxonómica y abundancia relativa de la fauna de Arthropoda}

El estudio se realizó en la franja costera de la región norte-centro de Chile, desde los $27^{\circ}$ a los $30^{\circ} \mathrm{S}$ (aproximadamente $450 \mathrm{~km}$ ). La composición taxonómica (a nivel ordinal) y las variaciones de abundancia relativa, especialmente de los artrópodos epígeos, se analizaron mediante trampas de intercepción instaladas en cuatro localidades (sitios en adelante, Fig. 1), designados de $\mathrm{N}$ a $\mathrm{S}$, como: ROD (Rodillo, 27 $7^{\circ} \mathrm{S}$; con $25 \mathrm{~mm}$ de precipitación anual histórica promedio), $\mathrm{CAB}$ (Carrizal Bajo, $28^{\circ} \mathrm{S}$; con $37 \mathrm{~mm}$ de precipitación), LCH (Los Choros, $29^{\circ} \mathrm{S}$; con 42 $\mathrm{mm}$ de precipitación), y LAG (Lagunillas, $30^{\circ}$ $\mathrm{S}$; con $110 \mathrm{~mm}$ de precipitación). Los sitios estudiados poseen suelos predominantemente arenosos, se encuentran en terrenos planos, a nivel del mar y libres de la condensación de

${ }^{1}$ CARMONA C, A JORQUERA, J CEPEDA-PIZARRO \& H VÁSQUEZ (2003) Artropodofauna de un ecotopo dunario costero del Parque Nacional Llanos de Challe (III Región de Atacama). XXV Congreso Nacional de Entomología, Talca, Chile, Libro de Resúmenes: 34.

2 PIZARRO-ARAYA J, V JEREZ \& J CEPEDA-PIZARRO (2004) Patrones distribucionales y áreas de endemismo para coleópteros epígeos del desierto costero del norte de Chile $\left(25^{\circ}-32^{\circ}\right.$ Lat S). XXVI Congreso Nacional de Entomología, Concepción, Chile, Libro de Resúmenes: 17. 


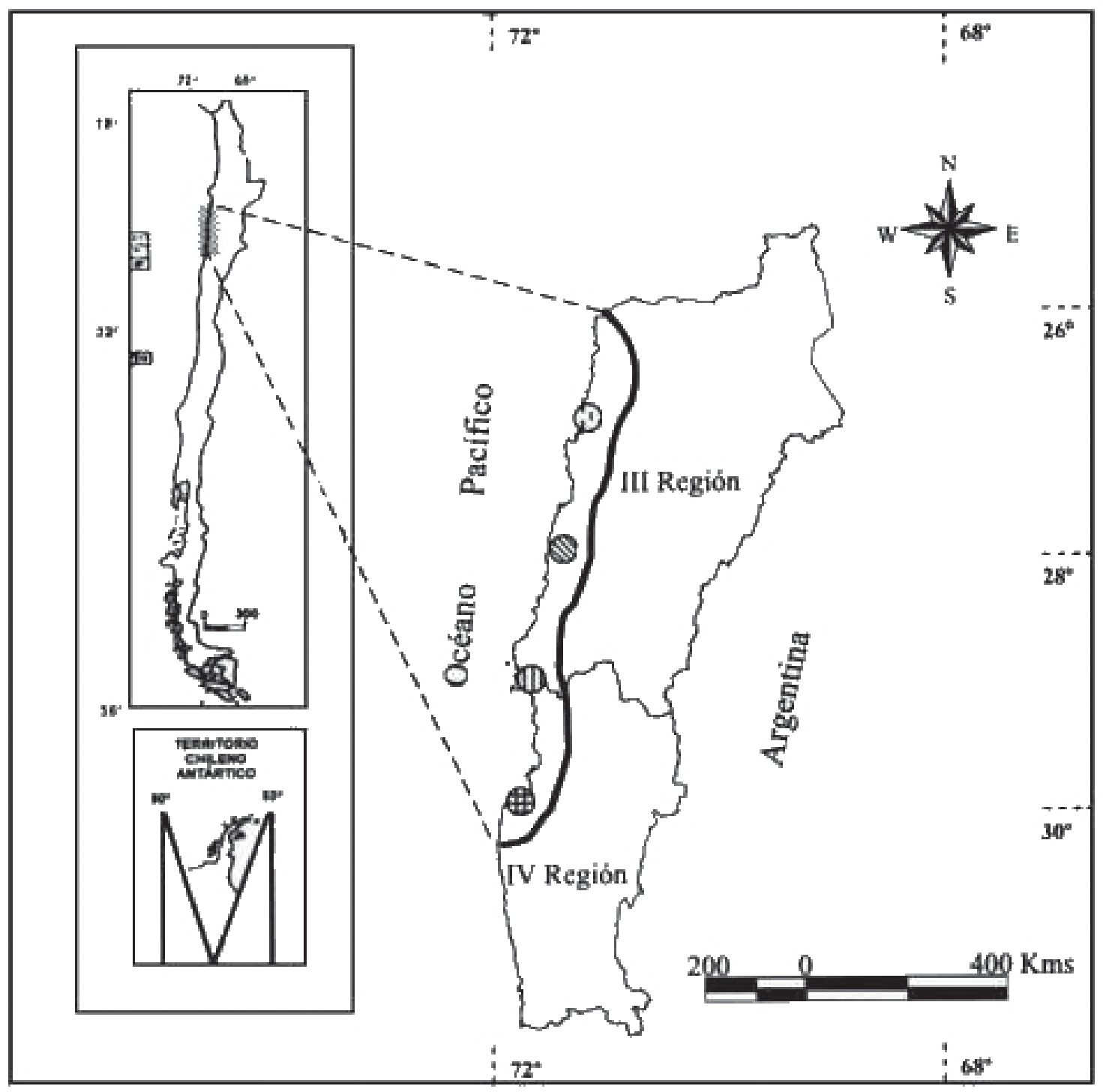

(i) Rodillo

Carrizal Bajo

(PN. Llanos de Challe)
(II) Los Choros

Lagunillas

Fig. 1: Ubicación geográfica de los cuatro sitios de estudio (Rodilllo, $27^{\circ} \mathrm{S}$; Carrizal Bajo, $28^{\circ} \mathrm{S}$; Los Choros, $29^{\circ} \mathrm{S}$; Lagunillas, $30^{\circ} \mathrm{S}$ ), en el desierto costero transicional de Chile.

Geographic location of four study sites (Rodilllo, $27^{\circ} \mathrm{S}$; Carrizal Bajo, $28^{\circ} \mathrm{S}$; Los Choros, $29^{\circ} \mathrm{S}$; Lagunillas, $30^{\circ} \mathrm{S}$ ), in the transitional-coastal desert of Chile. 
neblina, según descripción de Armesto et al. (1993). El tramo ha sido tipificado por Gajardo (1993) como perteneciente al desierto costero de Taltal (ROD), al desierto costero de Huasco $(\mathrm{CAB}$ y $\mathrm{LCH})$ y al matorral estepario costero (LAG). La descripción florística del área se encuentra en Armesto \& Vidiella (1993) y Armesto et al. (1993).

En cada uno de los sitios arriba mencionados se instaló una grilla de 4 x $5 \mathrm{~m}$ la que estuvo constituida por 20 trampas de intercepción dispuestas en líneas paralelas. La superficie efectiva de captura de la grilla fue $660 \mathrm{~m}^{2}$ (Péfaur \& Díaz 2000). Cada trampa consistió en un dispositivo formado por dos vasos plásticos dispuestos uno dentro del otro, con el vaso interior de fácil remoción. Las dimensiones de ambos vasos fueron 7,4 y 7,6 $\mathrm{cm}$ de diámetro x 10,2 y $12,0 \mathrm{~cm}$ de alto, respectivamente. El vaso interior fue llenado, hasta los dos tercios de su capacidad, con una mezcla de formalina (10\%), glicerina y agua con detergente doméstico (30\%), en una proporción de 3:1:6, respectivamente. Las trampas operaron en 1989 por 15 días cada mes, entre septiembre y diciembre, el período fenológico más favorable de la vegetación del área (Armesto et al. 1993, Vidiella et al. 1999). Los especímenes capturados fueron retirados, limpiados y conservados en alcohol glicerinado hasta el momento de su procesamiento y montaje. Para la nomenclatura de los taxa a nivel ordinal se sigue a Artigas (1994).

Composición taxonómica y variaciones en la abundancia relativa de tenebriónidos epígeos

El material de Tenebrionidae fue separado de los especímenes capturados según la metodología descrita en la sección anterior. La determinación de las especies de Tenebrionidae se hizo siguiendo a Peña $(1966,1980)$ y mediante comparación con material del Museo Nacional de Historia Natural (MNNC, Santiago) y del Instituto de Entomología, Universidad Metropolitana de Ciencias de la Educación (IEUMCE, Santiago). De los 19 géneros identificados, Nyctopetus, Hypselops y Geoborus fueron los elementos no epígeos s. str. Material referencial de estudio está depositado en la colección del laboratorio de entomología ecológica de la Universidad de La Serena (La Serena).

\section{RESULTADOS}

Composición taxonómica y variaciones de la abundancia relativa de la fauna de Arthropoda

Los insectos representaron el $\sim 95 \%$ del material capturado. El resto correspondió a arácnidos Solpugida, Scorpionida, Acari Parasitengonae y Araneae. Cinco órdenes dominaron el ensamble de Insecta; estos fueron Coleoptera (22,4 \% del total capturado), Diptera $(19,8 \%)$, Psocoptera $(13,4 \%)$, Collembola $(13,3 \%)$ e Hymenoptera $(11,6 \%)$. Las principales familias fueron: para Coleoptera, Tenebrionidae, Curculionidae, Meloidae y Staphylinidae; para Diptera, Agromyzidae, Tachinidae y Sciaridae; para Hymenoptera, Formicidae, Mutillidae y Braconidae; para Collembola, Onychiuridae y Entomobryidae. Órdenes con una menor representación porcentual fueron Lepidoptera $(4,7 \%$, con dominancia de Noctuidae, Hesperiidae y varias familias no identificadas de Microlepidoptera); Homoptera (3,7 \%, con dominancia de Cicadellidae, Psyllidae y Aphididae), y Orthoptera (3,0\%, representado principalmente por Gryllidae). Tanto por los taxa dominantes como por su contribución porcentual al total capturado, los sitios se diferenciaron entre sí. $\mathrm{CAB}$ fue el sitio que aportó la mayor cantidad de ejemplares $(41,3$ $\%)$, seguido por LAG $(25,8 \%)$ y LCH $(21,1 \%)$ Latitudinalmente según el total capturado por sitio, Psocoptera (70,2 \%) dominó en ROD $\left(27^{\circ}\right.$ S); Collembola (31,7 \%), Diptera $(21,4 \%)$ y Coleoptera $(15,5 \%)$ dominaron en CAB $\left(28^{\circ}\right.$ S); Diptera $(38,2 \%)$, Coleoptera $(29,6 \%)$ y Lepidoptera $(16,7 \%)$ lo hicieron en LCH $\left(29^{\circ}\right.$ S); Coleoptera $(35,8 \%)$, Hymenoptera $(21,6 \%)$ y Diptera $(10,3 \%)$ dominaron en LAG $\left(30^{\circ} \mathrm{S}\right)$. Mientras que para los taxa restantes no se observó una tendencia latitudinal, la abundancia relativa de Coleoptera aumentó de norte $(3,9 \%$ en ROD) a sur $(35,8 \%$ en LAG).

La Tabla 1 muestra la distribución espaciotemporal de la denso-actividad de los principales taxa, expresada como su contribución porcentual al total capturado por sitio por mes. En 1989, bajo una precipitación anual $\sim 44 \%$ inferior al promedio histórico, la denso-actividad máxima para todo el transecto se observó en noviembre $(\sim 31,2 \%$ del total capturado). Entre sitios, el mes más favorable 
fue, de norte a sur, septiembre para ROD, noviembre para CAB-LCH y diciembre para LAG. Se destaca que no fue posible estudiar la repuesta de la fauna de artrópodos de ROD en diciembre por destrucción de parte de las trampas. No obstante esta situación, ROD mostró una clara tendencia decreciente en su contribución numérica a lo largo del período estudiado (Tabla 1), por lo que su aporte a los recuentos de diciembre se consideró marginal.
Composición taxonómica y variaciones en la abundancia relativa de tenebriónidos epígeos

Tenebrionidae representó el $67 \%$ del total capturado de Coleoptera, con 14 géneros y 19 especies. La riqueza contabilizada en el período estudiado varió entre sitios (Tabla 2). El sitio más meridional y mésico del tramo (LAG) fue el más diverso (con 12 especies registradas); el sitio más septentrional y árido (ROD) fue el

TABLA 1

Contribución porcentual (mes por sitio) de los principales órdenes de Insecta y Arachnida habitantes del desierto costero transicional de Chile $\left(27-30^{\circ} \mathrm{S}\right)$. Método de trampeo: trampas de intercepción

Percent contribution (month per site) of main orders of Insecta and Arachnida inhabiting the transitional coastal desert of Chile $\left(27-30^{\circ} \mathrm{S}\right)$. Sampling device: pitfall trapping

\begin{tabular}{|c|c|c|c|c|c|c|c|c|c|c|c|c|c|c|c|c|c|}
\hline \multirow[b]{2}{*}{ Mes } & \multicolumn{17}{|c|}{ Taxon } \\
\hline & Sitio & CLL & ThY & ORT & PSO & HEM & HOM & THY & $\mathrm{COL}$ & DIP & HYM & NEU & SIP & LEP & LAR & ARA & $\mathrm{n}_{\text {capturado }}$ \\
\hline \multirow[t]{4}{*}{ Sep } & ROD & 0,9 & 0 & 0,2 & 73,7 & 0,9 & 1,8 & 0 & 3,9 & 0,5 & 10,0 & 0 & 0 & 1,8 & 3,3 & 3 & 2.232 \\
\hline & $\mathrm{CAB}$ & 26,7 & 0 & 1,8 & 6,8 & 0,3 & 1,7 & 0,2 & 2,4 & 40,9 & 7,6 & 0 & 0 & 4,3 & 0,3 & 7,0 & 1.332 \\
\hline & $\mathrm{LCH}$ & 0 & 0 & 0,9 & 1,3 & 0,3 & 2,0 & 0,1 & 8,5 & 65,3 & 4,0 & 0 & 0 & 16,1 & 0 & 1,5 & 1.155 \\
\hline & LAG & 0 & 0,5 & 5,6 & 8,7 & 2,2 & 24,0 & 5,6 & 23,0 & 15,7 & 8,1 & 0 & 0 & 0 & 1,9 & 4,7 & 973 \\
\hline \multirow[t]{4}{*}{ Oct } & ROD & 3,2 & 0 & 0,7 & 76,1 & 0,6 & 1,7 & 0 & 4,2 & 2,6 & 5,7 & 0 & 0 & 2,1 & 0,9 & 2,2 & 1.960 \\
\hline & $\mathrm{CAB}$ & 57,6 & 0 & 2,6 & 7,5 & 0,3 & 1,1 & 0,2 & 3,6 & 12,0 & 7,3 & 0 & 0 & 1,6 & 0,2 & 6,0 & 4.352 \\
\hline & $\mathrm{LCH}$ & 0 & 0 & 1,8 & 3,3 & 0,5 & 3,7 & 0,1 & 5,0 & 48,2 & 7,6 & 0 & 0 & 28,4 & 0 & 1,4 & 1.889 \\
\hline & LAG & 0 & 1,3 & 3,8 & 6,3 & 0,4 & 14,3 & 13,6 & 24,0 & 20,6 & 10,9 & 0 & 0,1 & 0 & 0,2 & 4,5 & 2.164 \\
\hline \multirow[t]{4}{*}{ Nov } & ROD & 0,7 & 0 & 15,0 & 24,4 & 0,2 & 1,7 & 0 & 3,1 & 1,2 & 21,5 & 0 & 0 & 1,7 & 21,7 & 8,8 & 414 \\
\hline & $\mathrm{CAB}$ & 35,3 & 0 & 3,1 & 6,9 & 0,3 & 1,5 & 0 & 16,0 & 17,0 & 10,7 & 0 & 0 & 0,3 & 0,3 & 8,6 & 6.363 \\
\hline & $\mathrm{LCH}$ & 0 & 0 & 2,8 & 0,7 & 0,2 & 4,7 & 0 & 37,2 & 32,9 & 3,0 & 0 & 0 & 16,7 & 0 & 1,8 & 3.023 \\
\hline & LAG & 0 & 0,4 & 1,4 & 4,5 & 0,3 & 2,8 & 0 & 60,0 & 7,6 & 14,0 & 3,0 & 0 & 3,1 & 0,7 & 2,2 & 2.426 \\
\hline \multirow[t]{3}{*}{ Dic } & $\mathrm{CAB}$ & 0,9 & 0 & 5,4 & 6,3 & 0,3 & 1,7 & 0,2 & 31,4 & 31,8 & 8,8 & 0 & 0 & 0,4 & 0 & 12,8 & 4.155 \\
\hline & $\mathrm{LCH}$ & 0 & 0 & 3,1 & 1,7 & 0,5 & 3,8 & 0 & 51,3 & 22,9 & 7,6 & 0 & 0 & 7,2 & 0,2 & 1,7 & 2.223 \\
\hline & LAG & 0 & 1,6 & 4,3 & 9,4 & 0,6 & 4,2 & 0 & 31,3 & 5,7 & 33,6 & 0 & 0 & 3,4 & 0,2 & 5,7 & 4.574 \\
\hline $\mathrm{n}_{\text {capturado }}$ & & 5.232 & 116 & 1.203 & 5.257 & 174 & 1.444 & 371 & 8.788 & 7.760 & 4.546 & 73 & 2 & 1.871 & 266 & 2.132 & 39.235 \\
\hline
\end{tabular}

Los valores en la hilera suman $100 \%$. Clave de abreviaciones: Sep $=$ septiembre $($ primavera inicial); Oct $=$ octubre (primavera); Nov = noviembre $($ primavera $) ;$ Dic $=$ diciembre $($ primavera final), CLL = Collembola; ThY = Thysanura; ORT = Orthoptera PSO = Psocoptera HEM = Hemiptera $;$ HOM = Homoptera $;$ THY = Thysanoptera COL = Coleoptera $;$ DIP = Diptera; HYM = Hymenoptera; NEU = Neuroptera; SIP = Siphonaptera; LEP = Lepidoptera; LAR = larvas de Insecta; ARA $=$ Arachnida (in toto); $\mathrm{n}=$ número de especímenes capturados

The numbers in the row add $100 \%$. Key to abbreviations: Sep = September (early spring); Oct = October $($ spring); Nov = November (spring); Dic = December (late spring), CLL = Collembola; ThY = Thysanura; ORT = Orthoptera; PSO = Psocoptera; HEM = Hemiptera; HOM = Homoptera; THY = Thysanoptera; COL = Coleoptera; DIP = Diptera; HYM = Hymenoptera; NEU = Neuroptera; SIP = Siphonaptera; LEP = Lepidoptera; LAR = Insecta larvae; ARA = Arachnida (in toto); $\mathrm{n}=$ number of specimens captured 
menos diverso (cinco especies). Sin embargo, esta tendencia latitudinal se rompió en $\mathrm{CAB}$, sitio cercano al extremo árido del tramo estudiado, el cual aportó un registro de 11 especies (Tabla 2). El número de especies activas varió tanto espacial como temporalmente. En ROD, el número máximo de especies activas (tres) se observó en octubre y noviembre, con ninguna de ellas activa en diciembre; en $\mathrm{CAB}$, aumentó gradualmente de cuatro especies registradas activas en septiembre a siete especies en diciembre. En LCH, se observó un patrón similar al registrado en $\mathrm{CAB}$, excepto que en $\mathrm{LCH}$ el número de especies activas varió de tres y seis. En LAG, aumentó continuamente de una especie registrada activa en septiembre a 11 en diciembre (Fig. 2).

Gyriosomus Guérin-Méneville fue el género más abundante y diverso de Tenebrionidae (Tabla 2). Las especies numéricamente dominantes fueron $G$. luczotii Laporte y $G$. elongatus Waterhouse (52,9 y 20,6\%, respectivamente). No obstante, mientras $G$. luczotii dominó en LAG, G. elongatus lo hizo en LCH. Algunas especies mostraron rangos estrechos de distribución. En esta situación están G. elongatus, solamente registrada en $\mathrm{LCH}, G$. bridgesi Waterhouse y G. luczotii solamente registradas en LAG, G. kingi Reed, G. planicollis Gebien y G. curtisi Fairmaire registradas solo en $\mathrm{CAB}$.

TABLA 2

Importancia relativa (en porcentaje) de las especies epígeas de Tenebrionidae del desierto costero transicional de Chile $\left(27-30^{\circ} \mathrm{S}\right)$

Relative importance (in percentage) of epigean species of Tenebrionidae of the transitional coastal desert of Chile $\left(27-30^{\circ} \mathrm{S}\right)$

\begin{tabular}{|c|c|c|c|c|c|c|}
\hline \multirow[b]{2}{*}{ Especie } & \multicolumn{4}{|c|}{ Sitio } & \multicolumn{2}{|c|}{ Total capturado } \\
\hline & ROD & $\mathrm{CAB}$ & $\mathrm{LCH}$ & LAG & $\mathrm{n}$ & $\%$ \\
\hline Gyriosomus luczotii Laporte & 0 & 0 & 0 & 52,93 & 3118 & 52,93 \\
\hline Gyriosomus elongatus Waterhouse & 0 & 0 & 20,62 & 0 & 1214 & 20,62 \\
\hline Gyriosomus kingi Reed & 0 & 6,42 & 0 & 0 & 378 & 6,42 \\
\hline Gyriosomus hoppei (Gray) & 0 & 0 & 0 & 3,60 & 212 & 3,60 \\
\hline Gyriosomus planicollis Gebien & 0 & 2,96 & 0 & 0 & 174 & 2,96 \\
\hline Gyriosomus curtisi Fairmaire & 0,05 & 0,58 & 0 & 0 & 38 & 0,64 \\
\hline Praocis sp. & 0,03 & 1,41 & 2,30 & 0,45 & 246 & 4,18 \\
\hline Nyctopetus sp. & 0 & 0 & 0 & 3,91 & 231 & 3,91 \\
\hline Scotobius sp. & 0,06 & 0,95 & 0,67 & 0 & 99 & 1,68 \\
\hline Blapstinus sp. & 0,19 & 0 & 0 & 0,25 & 26 & 0,44 \\
\hline Diastoleus sp. & 0 & 0,16 & 0,25 & 0,03 & 26 & 0,44 \\
\hline Geoborus sp. & 0 & 0 & 0 & 0,44 & 26 & 0,44 \\
\hline Thinobatis sp. & 0 & 0 & 0,33 & 0 & 19 & 0,33 \\
\hline Psammetichus sp. & 0,03 & 0,14 & 0 & 0,14 & 18 & 0,31 \\
\hline Hypselops sp. & 0 & 0 & 0,14 & 0,11 & 15 & 0,25 \\
\hline Psectrascelis sp. & 0 & 0,22 & 0 & 0 & 13 & 0,22 \\
\hline Nycterinus sp. & 0 & 0,03 & 0 & 0,10 & 7 & 0,13 \\
\hline Entomochilus sp. & 0 & 0 & 0 & 0,11 & 7 & 0,11 \\
\hline Physogaster sp. & 0 & 0,02 & 0 & 0 & 2 & 0,02 \\
\hline Especie no determinada & 0 & 0,03 & 0 & 0,22 & 15 & 0,25 \\
\hline Total & 0,36 & 12,92 & 24,31 & 62,29 & 5.884 & 99,88 \\
\hline Número de especies por sitio & 5 & & 11 & 6 & 12 & 20 \\
\hline
\end{tabular}

Código para sitio: como en Tabla 1

Code for site: as in Table 1 


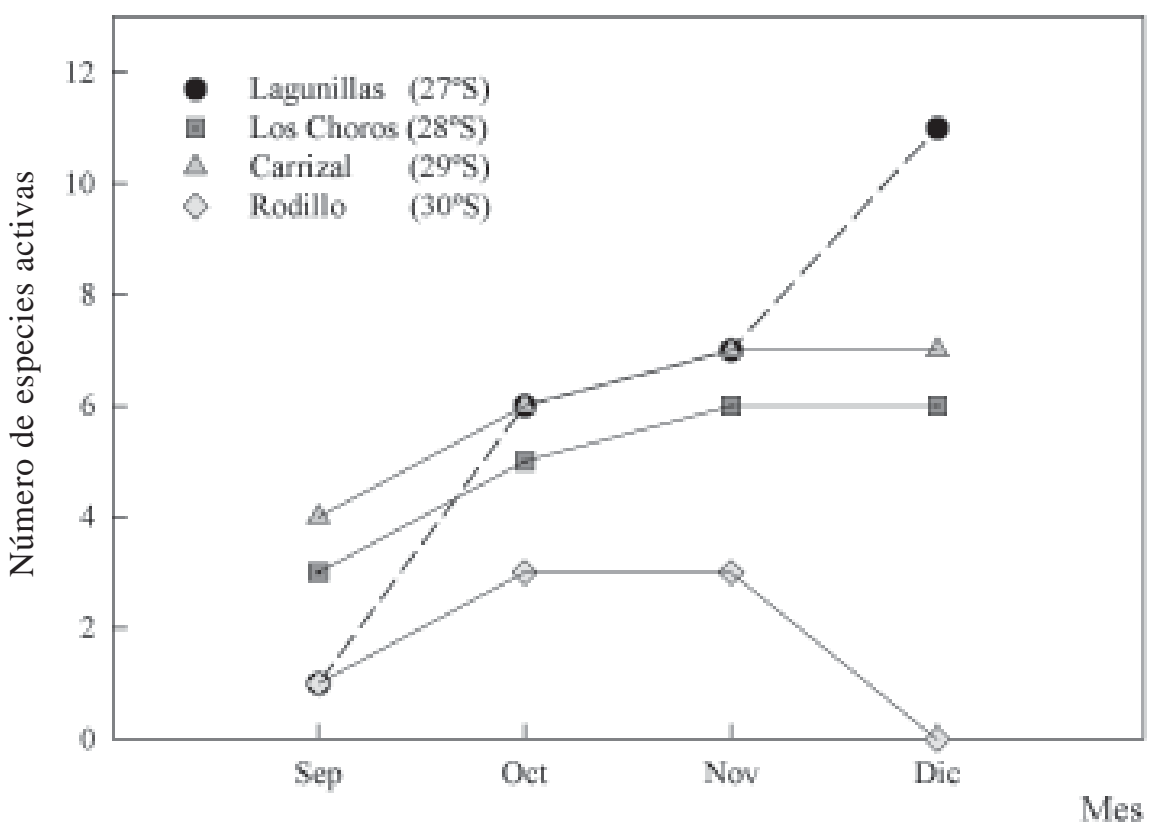

Fig. 2: Curvas de actividad de especies representativas de Tenebrionidae en cuatro sitios del desierto costero transicional de Chile, durante un año con precipitación anual (0-72 $\mathrm{mm})$ inferior al rango promedio histórico del área $(30-110 \mathrm{~mm})$.

Activity curves of representative species of Tenebrionidae in four sites of the transitional coastal desert of Chile, during a year with recorded rainfall $(0-72 \mathrm{~mm})$ below the range of historical mean for the area $(30-110 \mathrm{~mm})$.

\section{DISCUSIÓN}

El muestreo de artrópodos epígeos basado en trampas de intercepción, aunque bastante usado, exige tener presente algunas limitaciones de la técnica. En primer lugar, las trampas de intercepción están principalmente dirigidas al ensamble de artrópodos caminadores (Greenslade 1964, Jensen \& Mestz 1977, de los Santos et al. 1982, Cepeda-Pizarro 1987, Southwood 1994, Péfaur \& Díaz 2000, de los Santos et al. 2000, Work et al. 2002, Koivula et al. 2003); aun cuando se reconoce que en ecosistemas desérticos se puede lograr con ellas capturas representativas de especies de insectos voladores y fitófagos (Pietruszka 1980). En segundo lugar, la eficiencia de las trampas depende tanto del efecto que tienen algunas variables atmosféricas sobre la actividad de los individuos (e.g., temperatura del aire), como de la presencia de barreras que limiten su libre desplazamiento (e.g., mantillo herbáceo u orgánico) (Greenslade 1964, Gist \& Crossley 1973, Hinds \& Rickard 1973, Adis 1979). Por lo anterior, los resultados numéricos de las capturas se entienden frecuentemente como expresión de "denso-actividad" en lugar de densidad a solas (Luff 1975, Niemelä 1992, Southwood 1994, de los Santos et al. 2000). No obstante las deficiencias arriba mencionadas, las trampas de intercepción han resultado satisfactorias en el estudio de los ensambles de artrópodos de ambientes áridos, particularmente Tenebrionidae (Rickard \& Haverfield 1965, Ahearn 1971, Gist \& Crossley 1973, Thomas \& Sleeper 1977, Pietruszka 1980, Cepeda-Pizarro 1989, de los Santos et al. 2000, 2002a, 2002b).

Respecto de la presente investigación, problemas adicionales surgieron con el elevado número de especímenes capturados y con el estado del conocimiento de la diversidad taxonómica específica para esta región del país (Solervicens 1995, Simonetti 2000).

En el transecto estudiado, con una precipitación anual histórica que varía de norte a sur entre 25-110 mm (Toledo \& Zapater 1989), se capturaron 39.235 individuos en cuatro muestreos de 15 días de duración realizados entre septiembre y diciembre de un año con precipitación $44 \%$ bajo el promedio. 
Una fracción de ellos correspondió a elementos voladores (e.g., Noctuidae, Hesperiidae, Agromyzidae, Tachinidae, Sciaridae) y fitófagos (e.g., Cicadellidae, Psyllidae, Aphididae). Aunque no son epígeos sensu stricto Wallwork (1970, 1982), estos taxa se incluyeron en este trabajo siguiendo a Pietruszka (1980). Su registro constituye un dato relevante para comprender la importancia, temporal o espacial, que ellos pueden adquirir en el ecosistema examinado, especialmente cuando operan condiciones ambientales eventuales; por ejemplo, incursiones de El Niño Oscilación del Sur (Jaksic 1998, 2001, 2002³, Gutiérrez et al. 2000a, 2000b, Holmgreen et al. 2001).

De los grupos principales de Insecta registrados en los cuatro sitios estudiados, solo Coleoptera mostró una variación latitudinal de su abundancia, aumentando su presencia de norte a sur. Esta variación estaría relacionada con el aumento de la diversidad estructural de la vegetación documentada por Armesto et al. (1993), en concordancia a lo observado por Marquet et al. (1998), Rau et al. (1998), Spotorno et al. (1998) y Veloso \& Núñez (1998) en el tramo $21-26^{\circ} \mathrm{S}$. La abundancia relativa del resto de los taxa varió independientemente de la posición latitudinal del sitio, en aparente relación con las características edáficas, vegetacionales y de precipitación del año de estudio. Así, los elementos fitófagos, representados principalmente por Thysanoptera y Homoptera, mostraron mayores niveles de abundancia en el extremo meridional del transecto (LAG, matorral estepario costero, Gajardo 1993). Las condiciones más mésicas, la mayor cobertura vegetal (sobre $60 \%$ ) y la dominancia de malezas introducidas, características documentadas por Armesto \& Vidiella (1993), podrían ser responsables de la mayor abundancia de estos grupos en LAG. La distribución de los elementos fitófagos encontrada en este trabajo concuerda, en general, a lo comunicado por Rau et al. (1998) y Spotorno et al. (1998) respecto de artrópodos asociados al follaje de plantas vasculares del sector costero del tramo $21-26^{\circ} \mathrm{S}$. Los elementos

\footnotetext{
3 JAKSIC FM (2002) Efectos ecológicos de El Niño en ecosistemas terrestres de Sudamérica. XLV Reunión Anual de la Sociedad de Biología de Chile, Puyehue. Biological Research 35: R12.
}

voladores, representados principalmente por Diptera y Lepidoptera, alcanzaron valores porcentuales más altos en el tramo intermedio del transecto (i.e., $\mathrm{LCH}$ y $\mathrm{CAB}$ ). Se carece, de momento, de una explicación respecto de LCH (desierto costero de Huasco, Gajardo 1993). Por un lado, esta abundancia relativa pudiera estar relacionada con agricultura predial que se está instalando en terrenos de secano cercanos al sitio de estudio; pero pudiera también deberse a peculiaridades florísticas propias del lugar tal como ocurre con $\mathrm{CAB}$ (e.g., abundancia de anuales y geófitas nativas) (Armesto et al. 1993, Vidiella et al. 1999). La dominancia de Diptera y Lepidoptera en CAB podría estar asociada a las peculiaridades florísticas del sitio, particularmente en su papel como agentes polinizadores (Cepeda et al. 19964, Vidiella et al. 1999). De los elementos edáficos registrados en el transecto, los colémbolos son artrópodos que muestran preferencias por ambientes húmedos (Butcher et al. 1971, Wallwork 1970, 1982, Cepeda-Pizarro \& Whitford 1989, CepedaPizarro et al. 1996). Sin embargo, la dominancia de Collembola registrada en $\mathrm{CAB}$ en este estudio no se ajustó a la gradiente de precipitación del transecto, ya que el sitio se encuentra cerca del extremo xérico de este. Este resultado estaría indicando condiciones edáficas particulares de $\mathrm{CAB}$, derivadas tal vez de una mejor capacidad para conservar la humedad estacional, al efecto humectante de neblinas marinas o a las características florísticas del sector según descritas por Armesto et al. (1993) y Vidiella et al. (1999). Psocoptera, aunque registrado en todo el transecto, mostró una marcada preferencia por ROD (desierto costero de Taltal, Gajardo 1993), el extremo xérico del gradiente. No existe por el momento una explicación para la abundancia de Psocoptera en ROD; no obstante, su dominancia estaría indicando la existencia de especies bien adaptadas a hábitats xéricos, tal como ha sido sugerido por Camousseight \& New (1994). Los resultados sugieren la existencia de un conjunto de especies adaptadas a las condiciones xéricas del extremo septentrional del transecto.

${ }^{4}$ CEPEdA JG, H VÁSQUEZ \& G ARANCIO (1996) Asociación recurso floral/artrópodos en una comunidad desértica del norte de Chile. XVIII Congreso Nacional de Entomología. Temuco, Chile, Libro de Resúmenes: 28. 
Aparentemente, la distribución espaciotemporal de la abundancia de los taxa de Arthropoda examinados en este trabajo es sitioespecífica, siguiendo el comportamiento fenológico de la vegetación del área, según ha sido descrito por Armesto et al. (1993), Armesto \& Vidiella (1993), Vidiella et al. (1999). Estos resultados muestran también que, mientras la fauna de artrópodos del extremo mésico inicia su actividad más tempranamente en la estación favorable, aquella del extremo xérico lo hace más tardíamente.

\section{Abundancia y diversidad de Tenebrionidae}

Para el sector semiárido de Chile $\left(30-31^{\circ} \mathrm{S}\right)$, Sáiz \& Vásquez (1980) han observado que, mientras la diversidad específica y la densidad de la coleóptero-fauna disminuyen de sur a norte, aumenta el tamaño (talla y/o peso) de los individuos. La importancia relativa de Carabidae y Staphylinidae disminuye, pero aumenta la de Tenebrionidae. Durante 1989 y para todo el transecto, Tenebrionidae constituyó aproximadamente el $67 \%$ del registro de Coleoptera y el $15 \%$ del total capturado (39.235 especímenes). La contribución porcentual de cada sitio al total de tenebriónidos capturados mostró una tendencia positiva con el promedio anual de precipitación de los sitios del transecto. Por ejemplo, de norte a sur, ROD $(24,5 \mathrm{~mm}$ de precipitación registrada en el año) aportó el $0,36 \%$; CAB $(36,8 \mathrm{~mm})$, el $12,9 \%$; LCH (41,9 mm), el $24,3 \%$, y LAG $(106,0 \mathrm{~mm})$, el $62,3 \%$.

Los tenebriónidos del litoral costero del tramo $21-26^{\circ} \mathrm{S}$ (Antofagasta, II Región) están representados, en su sección meridional, por siete géneros y 18 especies; en la sección septentrional, por 12 géneros y 23 especies (Jerez 2000). Para el tramo $27-30^{\circ} \mathrm{S}$ examinado en este trabajo, se documenta la presencia de 14 géneros y 20 especies de Tenebrionidae, siendo Gyriosomus el género más diverso y abundante. Se destaca la ausencia de representantes del género Gyriosomus al norte de $\operatorname{los} 24^{\circ} \mathrm{S}$ y la distribución circunscrita al desierto costero de Taltal $\left(24-26^{\circ} \mathrm{S}\right)$ de las especies G. angustus Philippi y G. curtisi Fairmare (Pizarro-Araya \& Jerez 2004). De las especies de Gyriosomus reportadas en este estudio, solo G. curtisi se extiende hacia el norte del desierto costero transicional. Las especies numéricamente dominantes en el tramo y año estudiado (1989) fueron $G$. luczotii y $G$. elongatus. La distribución latitudinal de la riqueza de especies de Gyriosomus es sitio-específica en el desierto transicional de Chile. Hasta el momento, el género está representado por cuatro especies en el desierto costero de Taltal, 15 especies en el desierto costero de Huasco, cuatro especies en el desierto florido de Los Llanos, tres especies en el desierto florido de las serranías, y 10 especies en el matorral estepario costero. En este sentido, la distribución latitudinal de Gyriosomus en el tramo $21-30^{\circ} \mathrm{S}$ apoya la idea enunciada por algunos autores (e.g., Solervicens 1995, Jerez 2000, Pizarro-Araya \& Jerez 2004) que especies con individuos poco vágiles indicarían diferentes grados de diversidad y endemismo local, como podría ser el caso del sitio $\mathrm{CAB}$ (Pizarro-Araya et al. 2004²), en aparente concordancia con las características de la flora (Armesto et al. 1993) o con una mejor oferta de recursos alimentarios de calidad, tal como ha sido sugerido por Rau et al. (1998) y Spotorno et al. (1998) para los elementos entomológicos del transecto 21-26 $\mathrm{S}$. De acuerdo a observaciones de terreno y laboratorio efectuadas por uno de nosotros (HV), algunas especies de geófitas y anuales son intensivamente forrajeadas en terreno por los adultos de las especies de Gyriosomus (PizarroAraya \& Flores 2004).

Una adecuada interpretación de las variaciones en abundancia de la fauna de tenebriónidos epígeos depende de una clara comprensión de la dinámica vegetacional del sistema (Crawford 1988b, Rogers et al. 1988, Rau et al. 1998, Spotorno et al. 1998); de la acción de variables meteorológicas y edáficas (Ahearn 1971, Hinds \& Rickard 1973, Whicker \& Tracy 1987, Parmenter et al. 1989a, Reddy \& Venkataiah 1990, de los Santos et al. 2002a), y de las peculiaridades fisiológicas y de desarrollo de las especies que componen el ensamble (Knor 1975, Allsopp 1980, de los Santos et al. 1988, 2000, 2002b, Deslippe et al. 2001). Diversos autores, entre ellos Hinds \& Rickard (1973), Whicker \& Tracy (1987), de los Santos et al. (1988, 2002b), Parmenter et al. (1989b), Reddy \& Venkataiah (1990), han enfatizado el papel de la temperatura y la humedad del suelo sobre la abundancia de los 
tenebriónidos epígeos de los ecosistemas áridos y semiáridos. En el caso de este estudio, dado la condición de desierto costero templado (Rundel \& Mahu 1976, Rundel et al. 1991), es más probable que sean las variaciones hídricas inter e intraanuales, en lugar de las térmicas, las que jueguen un papel significativo en el desencadenamiento de las respuestas de desarrollo y reactivación de las especies de Tenebrionidae. Por otro lado, estudios sobre los requerimientos hídricos y patrones fenológicos de la vegetación del transecto (Armesto \& Vidiella 1993, Armesto et al. 1993, Vidiella et al. 1999) permiten suponer una relación entre esta y los patrones fenológicos de los componentes herbívoros de la comunidad. Estos autores han documentado la existencia de estrategias vitales de la flora acopladas con los patrones de disponibilidad de humedad edáfica. Por ejemplo, lluvias superiores a $20 \mathrm{~mm}$ que ocurran a fines de invierno rápidamente desencadenan la germinación, el crecimiento vegetativo y la floración de anuales y geófitas. Estos procesos determinan que tanto el número de especies activas como la cobertura de la vegetación muestren cambios abruptos durante la estación de crecimiento (septiembrediciembre), con máximos alrededor de octubrenoviembre, y declinación abrupta hacia enero. Los resultados encontrados en este trabajo indican que Tenebrionidae podría tener estrategias similares en respuestas a los eventos lluviosos que aseguren una adecuada disponibilidad de recursos alimentarios.

Es probable, según observaciones de terreno y laboratorio llevadas a cabo por nosotros, que la mayoría de las especies que conforman el ensamble se caracterice por una elevada fertilidad de los adultos y viabilidad de los huevos con un ciclo univoltino, con el último estadio larvario en diapausa; con el posterior paso a pupa y la eclosión de esta a finales del invierno o inicios de primavera; maduración rápida de los ovarios en primavera y ovipostura durante primavera-verano. Estos procesos, sin embargo, estarían en alguna forma regulados por la disponibilidad de agua, particularmente por su influencia sobre la fenología de la vegetación según comentado más arriba y la ecofisiología de los adultos, según ha sido documentado por Deslippe et al. (2001) y de los Santos et al. (2002a) para especies de Tenebrionidae de ecosistemas áridos de otras latitudes.

\section{AGRADECIMIENTOS}

Agradecemos los comentarios y sugerencias hechas al manuscrito por Julio Gutiérrez (Universidad de La Serena) y Viviane Jerez (Universidad de Concepción). El apoyo taxonómico en Tenebrionidae fue proporcionado por Mario Elgueta (Museo Nacional de Historia Natural, Santiago). Dos revisores anónimos y el editor de la revista sugirieron valiosas modificaciones editoriales al manuscrito, las que enriquecieron la versión final de este. El financiamiento fue proporcionado por la Universidad de La Serena (Dirección de Investigación y Desarrollo), subprograma de Investigación en ecosistemas áridos y semiáridos.

\section{LITERATURA CITADA}

ADAMOPOULOU C \& A LEGAKIS (2002) Diet of a lacertid lizard (Podarcis milensis) in an insular dune ecosystem. Israel Journal of Zoology 48: 207219.

ADIS J (1979) Problems of interpreting arthropods sampling with pitfall traps. Zoologischer Anzeiger 202: $177-184$.

AHEARN GA (1971) Ecological factors affecting population sampling of desert tenebrionid beetles. American Midland Naturalist 86: 385-406.

ALLSOPP PG (1980) The biology of false wireworms and their adults (soil-inhabiting Tenebrionidae) (Coleoptera): a review. Bulletin of Entomological Research 70: 343-379.

ARMESTO JJ \& PE VIDIELLA (1993) Plant life forms and biogeographic relations of the flora of Lagunillas $\left(30^{\circ} \mathrm{S}\right)$ in the fog-free pacific coastal desert. Annals of the Missouri Botanical Garden 80: 499-511.

ARMESTO JJ, PE VIDIELLA \& JR GUTIÉRREZ (1993) Plant communities of the fog-free coastal desert of Chile: plant strategies in a fluctuating environment. Revista Chilena de Historia Natural 66: 271-282.

ARTIGAS JN (1994) Entomología económica: insectos de interés agrícola, forestal, médico y veterinario. Ediciones Universidad de Concepción. Concepción, Chile. Volumen 1: v + 1126 pp.; Volumen 2: v + 943 pp.

AYAL Y \& O MERKL (1994) Spatial and temporal distribution of tenebrionid species (Coleoptera) in the Negev highlands, Israel. Journal of Arid Environments 27: 347-361.

BUTCHER JW, R SNIDER \& RJ SNIDER (1971) Bioecology of edaphic collembola and acarina. Annual Review of Entomology 16: 249-288.

CAMOUSSEIGHT A \& TR NEW (1994) Introducción a los insectos del orden Psocoptera en Chile. Museo Nacional de Historia Natural, Publicación Ocasional (Chile) 49: 1-26.

CARPANETO GM \& S FATTORINI (2001) Spatial and seasonal organisation of a darkling beetle (Coleoptera, Tenebrionidae) community inhabiting 
a Mediterranean coastal dune system. Italian Journal of Zoology 68: 207-214.

CARPANETO GM \& S FATTORINI (2003) Seasonal occurrence and habitat distribution of tenebrionid beetles inhabiting a Mediterranean coastal dune (Circeo National Park, Italy). Revue d' Ecologie-La Terre et la Vie 58: 293-306.

CEPEDA-PIZARRO JG (1987) Respuesta de los adultos de Gyriosomus luczotii (Coleoptera: Tenebrionidae) a las trampas de intercepción en un ecosistema árido-costero del norte de Chile. Folia Entomológica Mexicana 7: 89-99.

CEPEDA-PIZARRO JG (1989) Actividad temporal de tenebriónidos epígeos (Coleoptera) y su relación con la vegetación arbustiva en un ecosistema árido de Chile. Revista Chilena de Historia Natural 62: 115-125.

CEPEDA-PIZARRO JG (1995) Síntesis ecológica del desierto costero peruano-chileno. Investigación y Desarrollo (Chile) 2: 4-13.

CEPEDA-PIZARRO JG \& WG WHITFORD (1989) The relationships between abiotic factors and the abundance patterns of soil microarthropods on a desert watershed. Pedobiologia 33: 79-86.

CEPEDA-PIZARRO JG, JR GUTIÉRREZ, L VALDERRAMA \& H VÁSQUEZ (1996) Phenology of the edaphic microarthropods in a Chilean coastal desert site and their response to water and nutrient amendments to the soil. Pedobiologia 40: 352-363.

CEPEDA-PIZARRO J, S VEGA, H VÁSQUEZ \& $\mathrm{M}$ ELGUETA (2003) Morfometría y dimorfismo sexual de Elasmoderus wagenknechti (Libermann) (Orthoptera: Tristiridae) en dos eventos de irrupción poblacional. Revista Chilena de Historia Natural 76: 417-435.

CHEN X, MB THOMPSON \& CR DICKMAN (2004) Energy density and its seasonal variation in desert beetles. Journal of Arid Environments 56: 559-567.

COWLING RM, PW RUNDEL, BB LAMONT, MK ARROYO \& M ARIANOUTSOU (1996) Plant diversity in Mediterranean-climate regions. Trends in Ecology and Evolution 11: 362-366.

CRAWFORD CS (1979) Desert detritivores. A review of life history patterns and trophic roles. Journal of Arid Environments 2: 31-42.

CRAWFORD CS (1981) Biology of desert invertebrates. Springer-Verlag, New York, New York, USA. xvi + $314 \mathrm{pp}$.

CRAWFORD CS (1988a) Nutrition and habitat selection in desert detritivores. Journal of Arid Environments 14: 111-121.

CRAWFORD CS (1988b) Surface-active arthropods in a desert landscape: influences of microclimate, vegetation, and soil surface on assemblage structure. Pedobiologia 32: 373-385.

CRAWFORD CS, WP MACKAY \& JG CEPEDAPIZARRO (1993) Detritivores of the Chilean arid zone $\left(27-32^{\circ} \mathrm{S}\right)$ and the Namib desert: a preliminary comparison. Revista Chilena de Historia Natural 66: 283-289.

DAJOZ R (1984) Les coleópteres ténebrionides des deserts. Bulletin des Naturalistes Parisiens, Nouvelle Serie (France) 38: 26-57.

DE LOS SANTOS A, C MONTES \& L RAMÍREZ-DÍAZ (1982) Un nuevo diseño de trampa de caída para el estudio de poblaciones de coleópteros terrestres de superficie. Mediterránea Serie Biológica 6: 93-99.

DE LOS SANTOS A, C MONTES \& L RAMÍREZ (1988) Life histories of some darkling beetles (Coleoptera:
Tenebrionidae) in two Mediterranean ecosystems in the lower Guadalquivir (southwest, Spain). Environmental Entomology 17: 799-814.

DE LOS SANTOS A, LA GÓMEZ-GONZÁLEZ, C ALONSO, CD ARBELO \& JP de NICOLÁS (2000) Adaptative trends of darkling beetles (Col. Tenebrionidae) on environmental gradients on the island of Tenerife (Canary Islands). Journal of Arid Environments 45: 85-98.

DE LOS SANTOS A, EJ ALONSO, E HERNÁNDEZ \& AM PÉREZ (2002a) Environmental correlates of darkling beetles population size (Col. Tenebrionidae) on the Cañadas of Taide in Tenerife (Canary Islands). Journal of Arid Environments 50: 287-308.

DE LOS SANTOS A, JP de NICOLAS \& F FERRER (2002b) Habitat selection and assemblage structure of darkling beetles (Col. Tenebrionidae) along environmental gradients on the Island of Tenerife (Canary Islands). Journal of Arid Environments 52: 63-85.

DESLIPPE RJ, JR SALAZAR \& YL GUO (2001) A darkling beetle population in west Texas during the 1997-1998 El Niño. Journal of Arid Environments 49: 711-721.

DÍAZ JA \& M DÍAZ (1990) Estimas de tamaños y biomasas de artrópodos aplicables al estudio de la alimentación de vertebrados insectívoros. Doñana Acta Vertebrados (Spain) 17: 67-74.

FATTORINI S (2001) Temporal and spatial variations in darkling beetle predation by kestrels and other raptors in a Mediterranean urban area. Biología 56: 165-170.

FLORES GE (1998) Tenebrionidae. En: Morrone JJ \& S Coscarón (eds) Biodiversidad de artrópodos argentinos: una perspectiva biotaxonómica: 232-257. Ediciones Sur, Buenos Aires, Argentina. vii + 599 pp.

FORTUNA J (1938) Coleópteros de la región noroeste del territorio de La Pampa. Revista de la Sociedad Entomológica Argentina 10: 59-63.

FUENTES E \& C CAMPUSANO (1985) Pest outbreaks and rainfall in the semiarid-region of Chile. Journal of Arid Environments 8: 67-72.

GAJARDO R (1993) La vegetación natural de Chile. Editorial Universitaria, Santiago, Chile. $165 \mathrm{pp}$.

GASTON KJ (2000) Global patterns in biodiversity. Nature 405: 220-227.

GIST CS \& DA CROSSLEY (1973) A method for quantifying pitfall trapping. Environmental Entomology 25: 951-952.

GREENSLADE PJM (1964) Pitfall trapping as a method for studying populations of Carabidae (Coleoptera). Journal Animal Ecology 33: 301-310.

GUTIÉRREZ JR, G ARANCIO \& FM JAKSIC (2000a) Variation in vegetation and seed bank in a Chilean semi-arid community affected by ENSO 1997. Journal of Vegetation Science 11: 641-648.

GUTIÉRREZ JR, PL MESERVE \& FM JAKSIC (2000b) Efectos de la Corriente de El Niño sobre la biota terrestre de ecosistemas áridos de Sudamérica. En: Jiménez-Milón P, C Talavera-Delgado, L VillegasParedes, A Ortega-Paredes \& F VillasanteBenavides (eds) Memorias del IV Congreso Latinoamericano de Ecología: 91-94. Industria Gráfica Regentus, Arequipa, Perú.

HINDS WT \& WH RICKARD (1973) Correlations between climatological fluctuations and a population of Philolithus densicollis (Horn) (Col. Tenebrionidae). Journal Animal Ecology 42: 341-351. 
HOLMGREEN M, M SCHEFFER, E EXCURRA, JR GUTIÉRREZ \& GMJ MOHREN (2001) El Niño effects on the dynamics of terrestrial ecosystems. Trends in Ecology and Evolution 16: 89-94.

JAKSIC FM (1998) The multiple facets of El Niño/ Southern Oscillation in Chile. Revista Chilena de Historia Natural 71: 121-131.

JAKSIC FM (2001) Ecological effects of El Niño in terrestrial ecosystems of western South America. Ecography 24: 241-250.

JENSEN MJW \& JAJ MESTZ (1977) How many victims will a pitfall make? Acta Biotheoretica 28: 98-122.

JEREZ V (2000) Diversidad y patrones de distribución geográfica de insectos coleópteros en ecosistemas desérticos de la región de Antofagasta, Chile. Revista Chilena de Historia Natural 73: 79-92.

KNOR IB (1975) Life cycles of darkling beetles (Col Tenebrionidae) of Tuva. Soviet Journal Ecology 6 : 458-461.

KOIVULA M, DJ KOTZE, L HIISIVUORI \& H RITA (2003) Pitfall trap efficiency: do trap size, collecting fluid and vegetation structure matter? Entomologica Fennica 14: 1-14

KRASNOV B, D WARD \& G SHENBROT (1996) Body size and length variation in several species of darkling beetles (Coleoptera: Tenebrionidae) along a rainfall and altitudinal gradient in the Negev desert (Israel). Journal of Arid Environments 34: 477-489.

LUFF ML (1975) Some features influencing the efficiency of pitfall traps. Oecologia 19: 345-357.

MARCUZZI G (1951) Tenebrioni como indicatore del clima. Revista di Biologia di Perugia (Italy) 43: 399 437.

MARQUET PA, F BOZINOVIC, GA BRADSHAW, C CORNELIUS, H GONZÁLEZ, JR GUTIÉRREZ, E HAJEK, JA LAGOS, F LÓPEZ-CORTÉS, L NÚÑEZ, EF ROSELLO, C SANTORO, H SAMANIEGO, VG STANDEN, JC TORRESMURA \& FM JAKSIC (1998) Los ecosistemas del Desierto de Atacama y área andina adyacente en el norte de Chile. Revista Chilena de Historia Natura 71: 593-617.

NIEMELÄ J, JR SPENCE \& DH SPENCE (1992) Habitat associations and seasonal activity of ground-beetles (Coleoptera, Carabidae) in central Alberta. Canadian Entomology 124: 521-540.

ORGEAS J, E VIDAL \& P PONEL (2003) Colonial seabirds change beetle assemblages on a Mediterranean island. Ecoscience 10: 38-44.

PARMENTER RR \& JA MACMAHON (1984) Factors influencing the distribution and abundance of ground-dwelling beetles (Coleoptera) in a shrubsteppe ecosystem: the role of shrub architecture. Pedobiologia 26: 21-34.

PARMENTER RR \& JA MACMAHON (1988) Factors limiting populations of arid-land darkling beetles (Coleoptera: Tenebrionidae): predation by rodents. Environmental Entomology 17: 280-286.

PARMENTER RR, CA PARMENTER \& CD CHENEY (1989a) Factors influencing microhabitat partitioning among coexisting species of arid-land darkling beetles (Tenebrionidae): Behavioral response to vegetation architecture. Southwestern Naturalist 34: 319-329.

PARMENTER RR, CA PARMENTER \& CD CHENEY (1989b) Factors influencing microhabitat partitioning among coexisting species of arid-land darkling beetles (Tenebrionidae): temperature and water conservation. Journal of Arid Environments 17: 57-67.
PÉFAUR JE \& A DÍAZ (2000) Consideraciones sobre comunidades de animales epígeos en zonas semiáridas en Sudamérica. Memorias del IV Congreso Latinoamericano de Ecología. En: Jiménez-Milón P, C Talavera-Delgado, L Villegas-Paredes, A OrtegaParedes \& F Villasante-Benavides (eds) Memorias del IV Congreso Latinoamericano de Ecología. Ecología y desarrollo sostenible: Reto de la América Latina para el tercer milenio: 76-82. Industria Gráfica Regentus, Arequipa, Perú.

PEÑA LE (1959) Las vaquitas del desierto. Noticiero Mensual del Museo Nacional de Historia Natural (Chile) 37: 2

PEÑA LE (1966) Catálogo de los Tenebrionidae (Coleoptera) de Chile. Entomologische Arbeiten aus dem Museum George Frey (Alemania) 17: 397-453.

PEÑA LE (1980) Aporte al conocimiento de los tenebriónidos de América del Sur (Coleoptera: Tenebrionidae). Revista Chilena de Entomología 10: 37-59.

PIETRUSZKA RD (1980) Observations on seasonal variation in desert arthropods in central Nevada. Great Basin Naturalist 40: 292-297.

PIZARRO-ARAYA J \& GE FLORES (2004) Two new species of Gyriosomus Guérin-Méneville from Chilean coastal desert (Coleoptera: Tenebrionidae: Nycteliini). Journal of the New York Entomological Society 112: 121-126.

PIZARRO-ARAYA J \& V JEREZ (2004) Distribución geográfica del género Gyriosomus GuérinMéneville, 1834 (Coleoptera: Tenebrionidae): una aproximación biogeográfica. Revista Chilena de Historia Natural 77: 491-500.

RAU JR, C ZULETA, A GANZ, F SÁIZ, A CORTÉS, L YATES, AE SPOTORNO \& E COUVE (1998) Biodiversidad de artrópodos y vertebrados terrestres del norte grande de Chile. Revista Chilena de Historia Natural 71: 527-554.

REDDY MV \& B VENKATAIAH (1990) Seasonal abundance of soil-surface arthropods in relation to some meteorological and edaphic variables of the grassland and tree-planted areas in a tropical semiarid savannah. International Journal of Meteorology 34: 49-59.

RICKARD WH \& LE HAVERFIELD (1965) A pitfall trapping survey of darkling beetles in desert steppe vegetation. Ecology 46: 873-875.

ROGERS LE, NE WOODLEY, JK SHELDON \& PA BEEDLOW (1988) Diets of darkling beetles (Coleoptera: Tenebrionidae) within a shrub-steppe ecosystem. Annals of the Entomological Society of America 81: 782-791.

RUNDEL PW \& M MAHU (1976) Community structure and diversity in a coastal fog desert in northern Chile. Flora 165: 493-505.

RUNDEL PW, MO DILLON, B PALMA, HA MOONEY, SL GULMON \& JR EHLERINGER (1991) The phytogeography and ecology of the coastal Atacama and Peruvian deserts. Aliso 13: 1-49.

SÁIZ F \& E VÁSQUEZ (1980) Taxocenosis coleopterológicas epígeas en estepas de Chile semiárido. Anales del Museo de Historia Natural de Valparaíso (Chile) 13: 145-157.

SEMIDA FM, MS ABDEL-DAYEM, SM ZALAT \& FS GILBERT (2001) Habitat heterogeneity and altitudinal gradients in relation to beetle diversity in South Sinai, Egypt. Egyptian Journal of Biology 3: 137-146.

SHELDON JK \& LE ROGERS (1984) Seasonal and habitat distribution of tenebrionid beetles in shrub- 
steppe communities of the Hanford site in eastern Washington. Environmental Entomology 13: 214220.

SIMONETTI J (2000) Diversidad biológica. En: Brzovic PF \& M Romaggi (eds) Informe país: el estado de medio ambiente en Chile-1999: 177-200. Colección Sociedad Estado y Políticas Públicas, Centro de análisis de Políticas Públicas, Universidad de Chile, Santiago, Chile. vii +425 pp.

SOLERVICENS J (1995) Consideraciones generales sobre los insectos, el estado de su conocimiento y las colecciones. En: Simonetti JA, MTK Arroyo, A Spotorno \& EC Lozada (eds) Diversidad biológica de Chile: 198-210. Comisión Nacional de Investigación Científica y Tecnológica, Santiago, Chile.

SOUTHWOOD TRE (1994) Ecological methods: with particular reference to the study of insect populations. Chapman \& Hall, London, United Kingdom. vii + $391 \mathrm{pp}$.

SPOTORNO AE, C ZULETA, A GANTZ, F SÁIZ, J RAU, M ROSENMANN, A CORTÉS, G RUIZ, L YATES, E COUVE \& JC MARÍN (1998) Sistemática y adaptación de mamíferos, aves e insectos fitófagos de la Región de Antofagasta, Chile. Revista Chilena de Historia Natural 71: 501-526.

THOMAS DB \& EL SLEEPER (1977) The use of pitfall traps for estimating the abundance of arthropods with special reference to the Tenebrionidae (Coleoptera). Annals of the Entomological Society of America 70: 242-248.

THOMAS DB (1979) Patterns in the abundance of some tenebrionid beetles in the Mojave desert. Environmental Entomology 8: 568-574.

TOLEDO X \& E ZAPATER (1989) Geografía general y regional de Chile. Editorial Universitaria, Santiago, Chile. $251 \mathrm{pp}$

VELOSO A \& H NÚÑEZ (1998) Inventario de especies de fauna de la Región de Antofagasta (Chile) y recursos metodológicos para almacenar y analizar información de biodiversidad. Revista Chilena de Historia Natural 71: 555-569.

VIDIELLA PE, JJ ARMESTO \& JR GUTIÉRREZ (1999) Vegetation changes and sequential flowering after rain in the southern Atacama Desert. Journal of Arid Environments 43: 449-458.

WALLWORK JA (1970) Ecology of soil animals. McGraw-Hill, London, United Kingdom. vii +283 pp.

WALLWORK JA (1982) Desert soil fauna. Praeger Scientific, London, United Kingdom. x + 296 pp.

WHICKER AD \& CR TRACY (1987) Tenebrionid beetles in the shortgrass prairie: daily and seasonal patterns of activity and temperature. Ecological Entomology 12: 97-108.

WISE DA (1985) Negative correlation between numbers of a darkling beetle and a carabid predator. Southwestern Naturalist 30: 148-150.

WORK TT, CM BUDDLE, LM KORINUS \& JR SPENCE (2002) Pitfall trap size and capture of three taxa of litter-dwelling arthropods: implications for biodiversity studies. Environmental Entomology 31: 438-448. 